\title{
Water-cooled radiator design and optimization research for high power 3D-Sip system encapsulate
}

\author{
Jijun Zhang, Dejian Zhou* \& Tianshou Liang \\ School of Electronic and Mechanical Engineering, Guilin University of Electronic Technology, Guilin \\ 541004, China
}

Keywords: the finite element; Water-cooled radiator; Multi-objective optimization

\begin{abstract}
For high power package water-cooled radiator of 3D-Sip system and by using the finite element software ANSYS numerical simulation analysis of the geometrical parameters and fluid velocity affecting the performance of radiator cooling, the condition of air cooling water-cooled radiator fin thickness, spacing, and affect the performance of water cooled radiator cooling fluid flow curve is received. At the same time, water-cooled radiator outlet temperature of the inlet pressure and multi-objective optimization is based on the Pareto genetic algorithm. The results for high power 3D-Sip the design and system package of water-cooled radiator provides a certain basis.
\end{abstract}

\section{Introduction}

As the miniaturization of high power circuit module and the bad using environment, the cooling performance of they need stronger and stronger. The efficiency of forced water cooling is 20 times than the traditional cooling, it is a kind of good thermal design solutions[1]. According to the International Technology Roadmap for Semiconductors, single-chip power density of a high-performance system is expected to be higher than $100 \mathrm{~W} / \mathrm{cm} 2[2]$.In this paper, water cooling radiator for a specific high-power 3D-Sip (three-dimensional system in package) structure design, numerical simulation using ANSYS finite element techniques to analyze fluid flow, structural parameters of the cooling fins affect the fluid outlet temperature, at last based on genetic PARETO algorithms for fluid outlet temperature and fluid inlet pressure for multi-objective optimization, provide a reference for the design and optimization of high-power 3D-Sip water-cooled radiator system packaging.

\section{Problem description and model establishment}

\subsection{Problem description}

In this paper, a high power 3D-Sip packaging system for the design of water-cooled radiator, 15 $\mathrm{C}$ temperature drop was required. Referring to the technology of electronic radiator manual, it is assumed that the temperature of water inlet is $50 \mathrm{C}$, the initial size of outline dimension of water-cooled radiator is $44 \times 44 \times 10(\mathrm{~mm})$, in which the height of the heat dissipation fin is $8 \mathrm{~mm}$, water cavity is cubic and the size is $40 * 40 * 1.2(\mathrm{~mm})$, the outlet and the nozzle aperture is $0.8 \mathrm{~mm}$, fin thickness is $1.5 \mathrm{~mm}$, fin spacing is $3 \mathrm{~mm}$, the initial velocity of water on the entrance is $0.01 \mathrm{~m} / \mathrm{s}$, its physical parameters as shown in table 1.

Table 1: Material parameters

\begin{tabular}{|l|c|c|c|c|}
\hline Material & $\begin{array}{c}\text { density } \\
\left(\mathrm{kg} / \mathrm{m}^{3}\right)\end{array}$ & $\begin{array}{c}\text { conductivity } \\
(\mathrm{w} / \mathrm{m} \cdot \mathrm{k})\end{array}$ & $\begin{array}{c}\text { Specific heat } \\
(\mathrm{J} / \mathrm{kg} \cdot \mathrm{k})\end{array}$ & viscosity $\left(\mathrm{m}^{2} / \mathrm{s}\right)$ \\
\hline $\mathrm{Al}$ & 2880 & 240 & 90.64 & - \\
\hline Cooling medium & 997 & 0.613 & 4179 & $8.55 \mathrm{e}-4$ \\
\hline
\end{tabular}

\subsection{The establishment of simulation mode}

ANSYS is a large general finite element analysis software which gather structure, thermal, fluid, 
magnetic, acoustic physical fields at an organic whole, and it provides most of the CAD software interface, realizes data sharing and exchanging, but also provides the user with ANSYS as a platform for the development of a variety of tools [3].The model as shown in figure 1: this model to do the following assumptions: (1) set to turbulent flow, turbulence model judged by the Reynolds number $\operatorname{Re}=\rho v D h / \mu$, by $\operatorname{Re}<2300$ for laminar flow, instead of turbulence; $\rho$ is the medium density $(\mathrm{kg} / \mathrm{m} 3), \mathrm{v}$ is the fluid velocity $(\mathrm{m} / \mathrm{s}) ; \mu$ is the medium viscosity (pa, s);D is the water diameter [4].(2) the fluid is incompressible.(3) fluid and the flow of material parameters are constants.(4) the passage wall is smooth, and its thermal resistance is ignored.(5) all wall without sliding speed (zero).(6) only considers heat convection and heat conduction, the thermal radiation is ignored.

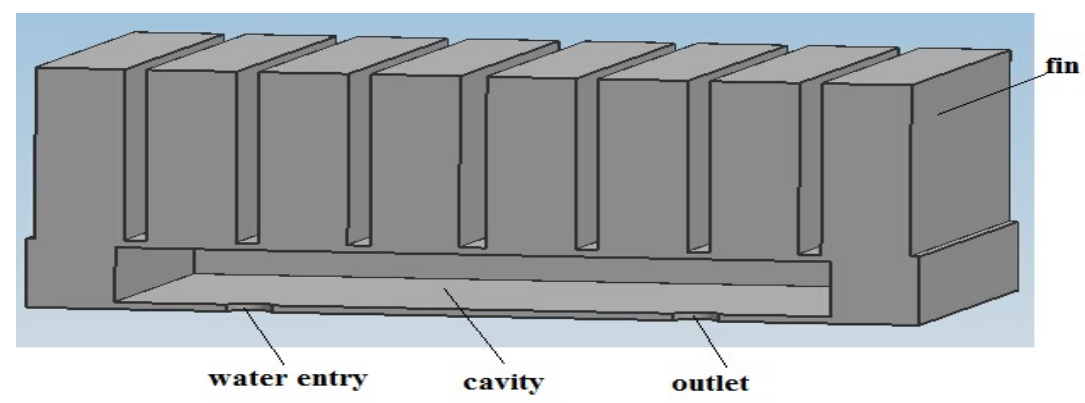

Fig.1: Water-cooled radiator simulation model

\subsection{The simulation results}

Post-processing by ANSYS simulation is showed in figure 2 and figure 3 results: the lowest temperature at the outlet $\mathrm{T}=33.51 \mathrm{C}$, entrance maximum pressure $\mathrm{P}=1.117 \mathrm{~Pa}$.

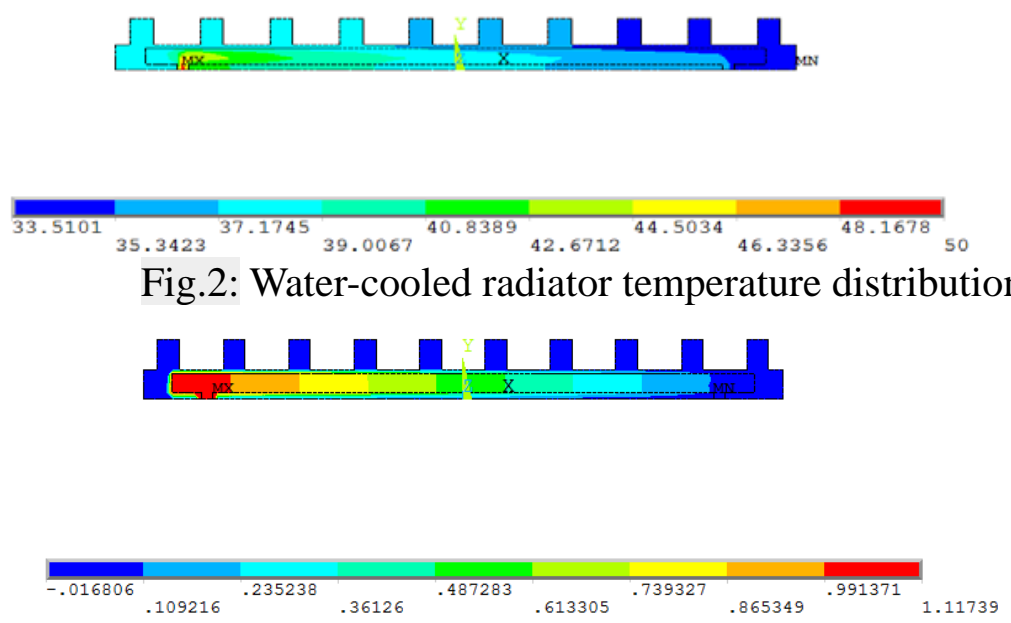

Fig.3: Water-cooled radiator pressure profile

\section{The analysis of the water-cooled radiator fluid outlet temperature}

Heat conduction and thermal convection is the main mode for water-cooled radiator heat transfer, the inlet velocity of fluid and air convection finned shapes are the important factors influencing the water cooled radiator. Under the condition of the inlet temperature must, the system heat dissipation effect is reflected directly by fluid outlet temperature. The structure of inlet velocity and fin parameters on the influence law of exit temperature is analyzed in this paper.

\subsection{The influence of outlet temperature by inlet velocity of fluid}

Air convection coefficient is generally constant, and the air forced convection is commonly 20 $100 \mathrm{w} /(\mathrm{m} 2 \cdot \mathrm{k})$. On account of the environment of the forced air cooling, the value of $50 \mathrm{w} /(\mathrm{m} 2 \cdot \mathrm{k})$ is selected. environment temperature is setting value $25 \mathrm{C}$, the inlet temperature is setting value 50 $\mathrm{C}$ and outlet pressure is setting value $0 \mathrm{~Pa}$. the equivalent inlet flow rate of inlet velocity and 6 kinds 
of inlet velocity is analyzed: $0.01,0.012,0.014,0.016,0.018,0.02(\mathrm{~m} / \mathrm{s})$, the entrance velocity of fluid outlet temperature (the actual is the average temperature on the fluid flow at the exit section) the influence of the curve is got according to the above condition, as shown in figure 4.

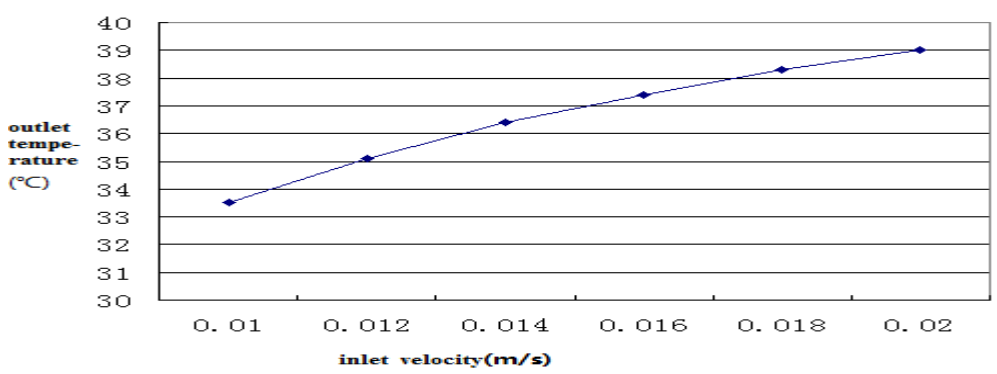

Fig. 4: The influence of the fluid velocity on the outlet temperature of the water cooled radiator curve

In the figure 4, we can see the faster the fluid inlet velocity, the higher the fluid outlet temperature. Because the fluid velocity is faster, fluid time is short laying in the cavity through the radiator and along with the fluid flow temperature is higher. In another context, as the inlet velocity increases gradually the temperature of the fluid flow radiator changes to flat.

\subsection{Fin structure parameters influences on the fluid outlet temperature}

Fin structure parameters are fin height, spacing and fin thickness. Because the water cooled radiator has certain restrictions on the space, so under the condition of the fin height direction unchanged, finned structure parameters is off for fin spacing and thickness of the rib two parameters. In this article, fin height is $1.5 \mathrm{~mm}$, the velocity is $0.01 \mathrm{~m} / \mathrm{s}$ in premise condition to study the fin spacing and fin thickness on the influence of fluid outlet temperature curve. As shown in figure 5, with the increase of fin spacing, exports also increases as the temperature. This is because with the increase of spacing, fin heat dissipation area is reduced, but the rate of change of temperature tends to smooth. Figure 6 reflects when fin number is 10, fin thickness influences of on the fluid outlet temperature curve.with the increase of fin thickness, air convection effect is weakened, so exports also increases as the temperature, but the rate of change of temperature is to increase trend.

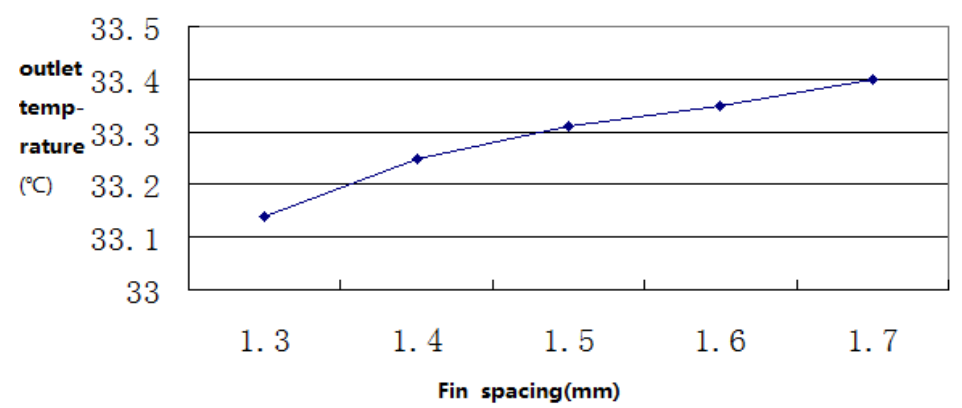

Fig. 5: The influence of fin spacing of outlet temperature curve

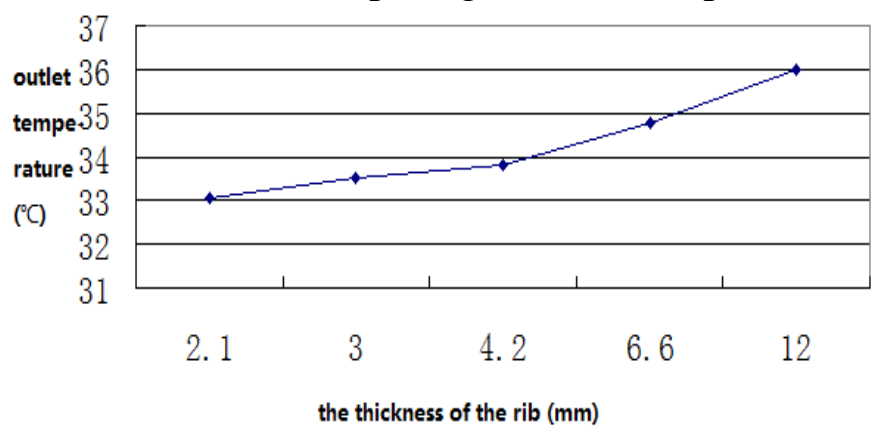

Fig. 6: The influence of fin thickness on the outlet temperature curve 


\section{Design of experiments and the relational model}

Due to the actual situation, water-cooled radiator of limited space, and inlet pressure affect the choice of the micro pump, so water cooled radiator in height must be under the premise of to study the number of fins, fin thickness, the velocity of three factors on the outlet temperature and inlet pressure, the relationship between establishing factors orthogonal test level such as in table 2.We can choose the orthogonal test table L9 (33).

Table 2: The orthogonal experiment factors - level

\begin{tabular}{|l|l|l|l|}
\hline factors & $\begin{array}{l}\text { Number of } \\
\text { fin }\end{array}$ & fin thickness & velocity \\
\hline Level 1 & 12 & 1.3 & 0.01 \\
\hline Level 2 & 10 & 1.5 & 0.016 \\
\hline Level 3 & 8 & 1.7 & 0.02 \\
\hline
\end{tabular}

The expressions of relation between fitting outlet temperature $\mathrm{T}$, fin number $\mathrm{N}$, fin thickness $\mathrm{D}$ and velocity $\mathrm{V}$ is fitted :

$T=25.96-0.28 * N+2.24 * D+762.2 * V-(N-10) 2 * 0.302-(N-10) *(D-1.5)$

$* 1.76+(D-1.5) 2 * 48.36-(N-10) *(V-0.015) 69.87-(D-1.5) *(V-0.015 * 852.24)$

The expressions of relation between fitting outlet Inlet pressure, fin number $\mathrm{N}$, fin thickness $\mathrm{D}$ and velocity $\mathrm{V}$ :

$P=-0.11-0.10 * N+0.64 * D+158.17 * V-(N-10) 2 * 0.085-(N-10) *(D-1.5)$

$* 0.47+(D-1.5) 2 * 2.61-(N-10) *(V-0.015) * 16.6-(E-1.5) *(V-0.015) * 245.55$. Pareto Algorithm to optimize

The core of multi-objective genetic algorithm is coordinating the relationship between different objective functions [5]. Based the conception of Pareto optimal solution, the method of calculating individual adaptation relies on the rank of non-inferior solution and corresponding selection operator to make the population evolve in the direction towards the Pareto optimal solutions in the process of optimization. The Indian scientist Deb based the NSGA and made some improvements, and presents NSGA- $\Pi$, a fast and non-inferior solution method: this defines crowding distance to estimate the solution density around a point instead of fitness sharing [6]. The function of Pareto is to make the solutions at a regional rather than a point solution, and the Pareto set filters to filter out non-inferior solution at every generation and eventually form a uniform set of Pareto solutions [7]. The initial control parameters of NSGA- II are set as follows: group size 60, the greatest evolutionary algebra 40 ,crossing probability 0.8 and mutation probability $0.2[8]$.

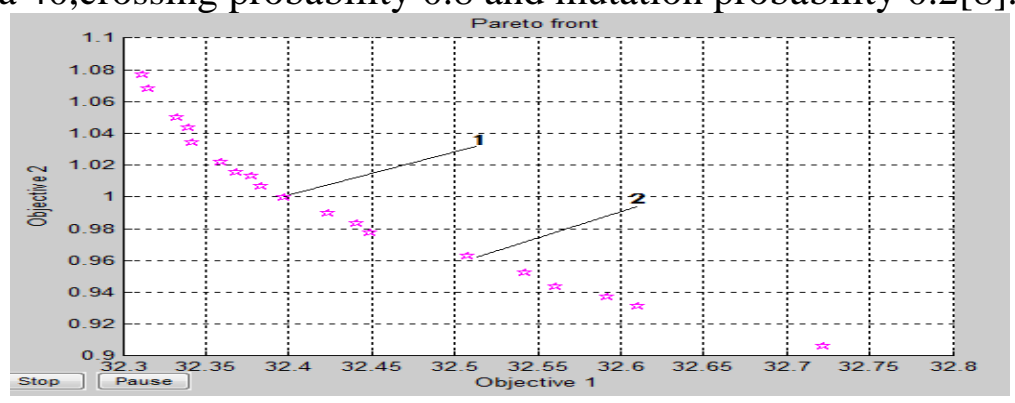

Fig.7: The optimized Pareto frontier

Figure7 shows the Pareto frontier of water cooling radiator outer temperature and inlet pressure after optimization. X-coordinate for the outlet temperature, y coordinates as the inlet pressure, red dot for optimized regulation of 21 groups of the solution set. If outlet temperature is the main factor, we can choose 1 point. If inlet pressure is the main factor, we can choose 2 point.

\section{Conclusion}

Though this article in view of the high power 3D-Sip system encapsulate water-cooled radiator design and optimization, we get the following main conclusions: (1) within the scope of a certain velocity, fluid outlet temperature with the increase of flow velocity increasing trends: the greater the 
flow velocity, the higher outlet temperature. (2) the fin height and velocity of certain premise condition, with the increase of fin spacing, the outlet temperature of the fluid as also increases, but the rate of change of temperature is tend to flat trend; With the increase of fin thickness, fluid outlet temperature as also increase, but the tendency of increase with the temperature change rate is tend to (3) based on the concept of Pareto optimal solution computation of individual fitness method, with the aid of the level of the Pareto solutions and the corresponding population selection operator in the optimization process between China and the DPRK Pareto optimal solution in the direction of evolution can quickly get optimal solution set, and have a good optimization effect. When to exit temperature as the main target can choose close to y reference points, when the inlet pressure as the main target can choose near the $\mathrm{x}$ axis reference point, as far as possible in the actual decision, finally also according to the actual situation in the choice of preference solution and multiple attribute decision making method to determine the final solution set.

\section{Acknowledgements}

This work was financially supported by Basic Scientific Research project-“*** Multiple Energy Loading Mechanism Analysis” and “***Function Layer Matching Rule and Multiple Energy Coordinated Control Method”

\section{References}

[1] Qing Wang,, Lianfa Yang, et al: Water cooled radiator simulation analysis of temperature field and thermal deformation. Fluid machinery. 39(1): 83-86.2011

[2] International Technology Roadmap for Semiconductors (2007)

[3] Longhan Xie,Xiang Li, et al: ANSYS FLOTRAN fluid and thermal analysis. Beijing: Electronic industry press,2012.

[4] Yang Xv,Shasha Dang, et al: The tutorial ANSYS11.0 flow field analysis of examples. Beijing: Mechanical industry press,2009.

[5] Shigang,Feng,Qian Ai. With elite strategy quickly non dominated sorting genetic algorithm in the application of multi-objective reactive power optimization. Journal of electrotechnics. (12): 146-151.2007

[6] Kalyanmoy Deb, Amrit Pratap, Sameer Agarwal \& Meyarivan, T.A., Fast and elitist multi-objective genetic algorithm: NSGA-II, IEEE Trans. Evol. Comp., 6(2), pp.182 197,2002.

[7] Anlin Wang, Xue Zhu,et al:Pareto Multi-objective genetic algorithm and its application in mechanical robust design. Mechanical design and research (12): 10-12.2007

[8] Chun Gong. Proficient in MATLAB optimization calculation. Beijing: Electronic industry press,2012. 